\title{
UNIFORM PERSISTENCE AND REPELLORS FOR MAPS
}

\author{
JOSEF HOFBAUER AND JOSEPH W.-H. SO
}

(Communicated by Kenneth R. Meyer)

\begin{abstract}
We establish conditions for an isolated invariant set $M$ of a map to be a repellor. The conditions are first formulated in terms of the stable set of $M$. They are then refined in two ways by considering (i) a Morse decomposition for $M$, and (ii) the invariantly connected components of the chain recurrent set of $M$. These results generalize and unify earlier persistence results.
\end{abstract}

\section{INTRODUCTION}

Let $\mathscr{X}$ be a metric space with metric $d$. A map $f: \mathscr{X} \rightarrow \mathscr{X}$ defines a discrete semi-dynamical system $T: \mathbf{Z}_{+} \times \mathscr{X} \rightarrow \mathscr{X}$ by $T(n, x)=f^{n}(x)$, where $\mathbf{Z}_{+}$denotes the set of non-negative integers and $f^{n}(x)$ denotes the $n$th iterate of $x$ under $f$. Let $\mathscr{Y}$ be a subspace of $\mathscr{X}$. We say that $f$ is uniformly persistent (with respect to $\mathscr{Y}$ ) if there exists $\eta>0$ such that for all $x \in \mathscr{X} \backslash \mathscr{Y}$, $\liminf _{n \rightarrow \infty} d\left(f^{n}(x), \mathscr{Y}\right)>\eta$. In applications to ecological equations, $\mathscr{X}$ will be the set of all possible states of the system and $\mathscr{Y}$ the set of extinction states. In that case, uniform persistence captures the idea of non-extinction of the system.

The object of this paper is to obtain criteria for uniform persistence. These criteria are formulated as conditions imposed on the global attractor $M$ of $\mathscr{Y}$. Hence, we first study uniform persistence on compact spaces $X(\xi \S 2$ and 3$)$ and apply these results to the general problem in $\S 4$. In particular, the persistence results in Freedman and So $[9,10]$ will be improved to include uniform persistence. The approach we use here is similar to the one used in Garay [11] and Hofbauer [14] for flows. It provides a more elegant approach to the persistence problem as it uses modern dynamical systems theory. Hence, this approach allows us to give simpler proofs for earlier persistence results based on average

Received by the editors November 7, 1988 and, in revised form, March 5, 1989.

1980 Mathematics Subject Classification (1985 Revision). Primary 58F12.

Key words and phrases. Discrete semi-dynamical systems, permanence, uniform persistence, repellor, isolated invariant set, Morse decomposition, chain recurrent set, basic sets, average Lyapunov functions, global attractor.

Research partially supported by the Natural Sciences and Engineering Research Council of Canada, grant number NSERC OGP36475. 
Lyapunov functions (cf. Hutson and Moran [17], Fonda [8], Hofbauer, Hutson and Jansen [15] and Hofbauer and Sigmund [16]).

\section{REPELLORS}

In this section and the next, the standing assumptions are:

(a) $X$ is a compact metric space,

(b) $f: X \rightarrow X$ is a continuous map, and

(c) $M \subset X$ is compact invariant, that is, $f(M)=M$.

For notation and terminology concerning discrete semi-dynamical systems not explained here, we refer the reader to LaSalle [18], Bhatia and Hajek [2] and Hale [12].

$M$ is said to be a repellor if there exists a neighborhood $U$ of $M$ such that for all $x \notin M$ there exists $n_{0}=n_{0}(x)>0$ satisfying $f^{n}(x) \notin U$ for all $n \geq n_{0}$. U is called a repellor neighborhood of $M$.

The following theorem is a special case of the Ura-Kimura theorem for maps (cf. Bhatia and Hajek [2, Chapter 9]).

Theorem 2.1. $M$ is a repellor if and only if

(1) $M$ is isolated, that is, there exists a closed neighborhood $U$ of $M(U$ is called an isolating neighborhood of $M$ ) such that $M$ is the largest invariant set in $U$, and

(2) $W^{s}(M) \subset M$, where $W^{s}(M):=\left\{x \in X: f^{n}(x) \rightarrow M\right.$ as $\left.n \rightarrow+\infty\right\}$ is the stable set of $M$.

Proof. The $\Rightarrow$ part is obvious. To show the converse, let $V$ be a compact isolating neighborhood of $M$. Then for each $x \notin M$, there exists arbitrarily large $n$ such that $f^{n}(x) \notin V$. (Otherwise, the $\omega$-limit set of $x, \Lambda^{+}(x):=$ $\bigcap_{n=0}^{\infty} \operatorname{cl}\left(\bigcup_{k=n}^{\infty} f^{k}(x)\right)$, will be an invariant subset of $V$. Since $V$ is an isolating neighborhood of $M, \Lambda^{+}(x) \subset M$. This contradicts (2).) Let $B=$ $\bigcup_{n=0}^{\infty} f^{n}(\operatorname{cl}(X \backslash V))$.

Claim. There exists $N \geq 0$ such that $B=\bigcup_{n=0}^{N} f^{n}(\operatorname{cl}(X \backslash V))$.

Indeed for all $x \in \operatorname{cl}(X \backslash V)$, there exists $n(x) \geq 1$ such that $f^{n(x)}(x) \in X \backslash V$. By compactness of $\operatorname{cl}(X \backslash V)$, there exists $N \geq 0$ such that for all $x \in \operatorname{cl}(X \backslash V)$, there exists $n, 1 \leq n \leq N$ such that $f^{n}(x) \in X \backslash V$, since $X \backslash V$ is open. This proves the claim.

Thus $B$ is compact, positively invariant (that is, $f(B) \subset B$ ) and disjoint from $M$ and $U=X \backslash B$ is a repellor neighborhood for $M$.

Remark. The set $A=\bigcap_{n=0}^{\infty} f^{n}(B)$ is an attractor, that is, there is a neighborhood $U$ of $A$ such that the $\omega$-limit set of $U, \Lambda^{+}(U):=\bigcap_{n=0}^{\infty} \operatorname{cl}\left(\bigcup_{k=n}^{\infty} f^{k}(U)\right)$, is $A$. It is called the dual attractor to the repellor $M$. Moreover, $W^{S}(A)=X \backslash M$. 
Corollary 2.2 (Fonda [8]). Let $X \backslash M$ be positively invariant. Then $M$ is a repellor if there exists a continuous function $P: X \rightarrow \mathbf{R}^{+}$satisfying the conditions:

(1) $P(x)=0$ for $x \in M$, and

(2) there exists a neighborhood $U$ of $M$ such that $\forall x \in U \backslash M \exists n>0$ such that $P\left(f^{n}(x)\right)>P(x)$.

Proof. Without loss of generality, we can assume $U$ is closed. Suppose $\exists x \notin$ $M$ with $\operatorname{cl}\left(\gamma^{+}(x)\right) \subset U, \gamma^{+}(x):=\left\{x, f(x), f^{2}(x), \ldots\right\} ;$ then $\exists y \in \operatorname{cl}\left(\gamma^{+}(x)\right)$ such that $P(y) \geq P(z)$ for all $z \in \operatorname{cl}\left(\gamma^{+}(x)\right)$. Hence, $y \notin M$ and $P(y) \geq$ $P\left(f^{n}(y)\right)$ for all $n \geq 0$. This contradicts (2). Therefore, $U$ is an isolating neighborhood of $M$ and $\forall x \notin M, \Lambda^{+}(x) \not \subset M$. Hence, by Theorem 2.1, $M$ is a repellor.

Corollary 2.3 (Hutson and Moran [17]). Let $X \backslash M$ be positively invariant. Then $M$ is a repellor if there exists a continuous function $P: X \rightarrow \mathbf{R}^{+}$such that

(1) $P(x)=0$ if and only if $x \in M$, and

(2) for all $x \in M, \sup \left\{\prod_{k=0}^{n-1} \psi\left(f^{k}(x)\right): n \geq 1\right\}>1$, where $\psi: X \rightarrow \mathbf{R}^{+}$is a continuous function with $P(f(x)) \geq \psi(x) P(x)$.

Proof. Let $U_{n}=\left\{x \in X: \psi(x) \cdots \psi\left(f^{n-1}(x)\right)>1\right\}$. Then $U_{n}$ is open and $U:=\bigcup_{n=1}^{\infty} U_{n} \supset M$. Therefore $P$ satisfies the conditions in Corollary 2.2 and $M$ is a repellor.

Remark. This shows that $M$ is a repellor if one can find a function $P$ as above such that $\psi>1$ on $\bigcup_{x \in M} \Lambda^{+}(x)$.

Let $\Gamma$ be a compact invariant set and let $x, y \in \Gamma$. An $\epsilon$-chain from $x$ to $y$ is a sequence of points $x_{0}=x, x_{1}, \ldots, x_{n}=y$ in $\Gamma$ with $d\left(f\left(x_{i}\right), x_{i+1}\right)<\epsilon$ for $i=0, \ldots, n-1$. We say that $x$ is chained to $y$ if for all $\epsilon>0$, there exists an $\epsilon$-chain from $x$ to $y$. A compact, invariant subset $\Lambda$ of $\Gamma$ is chain transitive if any two points $x$ and $y$ in $\Lambda$ are chained in $\Lambda$. If $x$ is chained to itself (in $\Gamma$ ), then $x$ is said to be a chain recurrent point and we write $x \in \mathscr{R}(\Gamma)$. Now, $x \notin \mathscr{R}(\Gamma)$ iff there exists an open set $U \subset \Gamma$ such that $f(\operatorname{cl}(U)) \subset U, x \notin U$ and $f(x) \in U$, or equivalently, iff there exists an attractor $A \subset \Gamma$ such that $x \in W^{s}(A) \backslash A$ (see Block and Franke [4, Theorem A]). Hence, $\mathscr{R}(\Gamma)=\bigcap\left(A \cup A^{*}\right)$, the intersection being taken over all attractorrepellor pairs $\left(A, A^{*}\right)$ of $\Gamma$.

The equivalence classes $C(x)=\{y \in \Gamma: x$ is chained to $y$ and $y$ is chained to $x$ \} are called the basic sets of $\Gamma . C(x)$ are chain transitive and they are the invariantly connected components of $\mathscr{R}(\Gamma)$ (see Conley [7] for flows and Akin [1] for maps). A compact invariant set is invariantly connected if it is not the disjoint union of two non-empty compact invariant sets.

Corollary 2.4 (Butler-McGehee lemma for maps (cf. Freedman and So [10])). Let $M$ be an isolated invariant set and let $x \in X$ be such that $\Lambda^{+}(x) \cap M \neq \varnothing$ and $\Lambda^{+}(x) \not \subset M$. Then $\Lambda^{+}(x) \cap\left(W^{s}(M) \backslash M\right) \neq \varnothing$. 
Proof. Let $X_{1}=\Lambda^{+}(x), M_{1}=\Lambda^{+}(x) \cap M, f_{1}=\left.f\right|_{X_{1}}$ and restrict ourselves to the dynamics of $f_{1}$ on $X_{1}$ for the moment. Suppose $\Lambda^{+}(x) \cap\left(W^{s}(M) \backslash M\right)=\varnothing$. Then $M_{1}$ is isolated invariant, $X_{1} \backslash M_{1}$ is positively invariant and $W^{s}\left(M_{1}\right) \subset$ $M_{1}$. By Theorem 2.1, $M_{1}$ is a (non-empty) repellor. Since $X_{1}$ is chain transitive (see Bowen [3]), it cannot have a non-trivial attractor-repellor pair. Thus, $M_{1}=X_{1}$ and this contradicts $\Lambda^{+}(x) \not \subset M$.

Remark. There is a characterization for attractors analogous to Theorem 2.1. It can be used to show that, under the hypotheses of Corollary 2.4, there exists a negative orbit in $\Lambda^{+}(x) \backslash M$ whose $\alpha$-limit is contained in $M$.

\section{MORSE DECOMPOSITIONS, BASIC SETS AND REPELLORS}

Let $M$ be a compact, invariant set. A finite collection $\left\{M_{1}, \ldots, M_{n}\right\}$ of compact invariant subsets of $M$ is called a Morse decomposition if the $M_{i}$ are pairwise disjoint and for each $x \in M \backslash \bigcup_{i=1}^{n} M_{i}$ there is an $i$ with $\Lambda^{+}(x) \subset M_{i}$ and for any negative orbit $x_{-}$through $x$ there is a $j>i$ with $\Lambda^{-}\left(x_{-}\right) \subset M_{j}$, where $\Lambda^{-}\left(x_{-}\right)$is the $\alpha$-limit set of $x_{-}$(cf. Mischaikow and Franzosa [19]).

Remark. It was shown in Freedman and So [10] that an acyclic covering, as introduced in Butler, Freedman and Waltman [5] and Butler and Waltman [6], is a Morse decomposition. The proof is based on the Butler-McGehee Lemma.

The following theorem is a refinement of Theorem 2.1 if a Morse decomposition of $M$ is given. It improves Theorem 3.3 of Freedman and So [10] as it yields uniform instead of strong persistence. Its proof shows that the existence of an acyclic covering for $M$ is equivalent to $M$ being isolated. The point is that one should choose an acyclic covering of $M$ so that the assumptions can be easily checked.

Theorem 3.1. Suppose $\left\{M_{1}, \ldots, M_{n}\right\}$ is a Morse decomposition of $M$ such that each $M_{i}$ is isolated in $X$. Then $M$ is a repellor if and only if $W^{s}\left(M_{i}\right) \subset M$ for each $i$.

Proof. The $\Rightarrow$ part is obvious. For the converse, we have to show that conditions (1) and (2) in Theorem 2.1 are satisfied.

First we will show (2). Let $x \notin M$. Suppose $\Lambda^{+}(x) \subset M$. Then $\Lambda^{+}(x) \subset$ $\mathscr{R}(M)$, the chain recurrent set of $M$. Since $\mathscr{R}(M) \subset \bigcup_{i=1}^{n} M_{i}$ and $\Lambda^{+}(x)$ is invariantly connected, there exists $i$ such that $\Lambda^{+}(x) \subset M_{i}$, contradicting $W^{s}\left(M_{i}\right) \subset M$.

To show (1), assume $M$ is not isolated. Then for every $n$, there exists $x_{n} \notin M$ with $\gamma^{+}\left(x_{n}\right) \subset\{x: d(x, M)<1 / n\}$, where $\gamma^{+}\left(x_{n}\right)$ is the positive orbit through $x_{n}$. The sets $\Omega_{n}=\Lambda^{+}\left(x_{n}\right)$ are compact, invariant and chain transitive. By (2), we know $\Omega_{n} \not \subset M$. A subsequence of $\left\{\Omega_{n}\right\}$, which we again denote by $\left\{\Omega_{n}\right\}$, converges in the Hausdorff metric to a compact set $\Omega \subset M$. Since $f\left(\Omega_{n}\right)$ converges to $f(\Omega), \Omega$ is invariant. By the argument in Garay [11, Proof of Theorem 2], $\Omega$ is also chain transitive. Therefore 
$\Omega \subset \mathscr{R}(M) \subset \bigcup_{i=1}^{n} M_{i}$. Since $\Omega$ is invariantly connected, $\Omega \subset M_{i}$ for some $i$. Hence any neighborhood of $M_{i}$ must contain some $\Omega_{n}$ for large $n$ and $M_{i}$ is not isolated.

The proof of Theorem 3.1 actually shows:

Theorem 3.2. $M$ is a repellor if and only if each basic set $\Lambda$ of $M$ satisfies

(1) $\Lambda$ is $M$-isolated, that is, there is a neighborhood $U$ of $\Lambda$ in $X$ such that if a complete orbit $\gamma(x) \subset U$ then $\gamma(x) \subset M$, and

(2) $W^{s}(\Lambda) \subset M$.

\section{UNIFORM PERSISTENCE}

Let $\mathscr{X}$ be a metric space with metric $d, f: \mathscr{X} \rightarrow \mathscr{X}$ be a continuous map and $\mathscr{Y} \subset \mathscr{X}$ is closed with $f(\mathscr{X} \backslash \mathscr{Y}) \subset \mathscr{X} \backslash \mathscr{Y}$.

We assume that $\mathscr{X}$ has a global attractor $X$, that is, $X$ is the maximal compact invariant subset of $\mathscr{X}$ and $d\left(f^{n}(x), X\right) \rightarrow 0$ as $n \rightarrow \infty$, for all $x \in \mathscr{X}$. This assumption is satisfied in many applications including maps, time-periodic ordinary differential equations, retarded delay equations as well as semi-linear parabolic equations (cf. Hale [12] and Hale and Waltman [13]).

Note that $\mathscr{Y}$ is in general not a positively invariant set. Let $M$ be the maximal compact invariant set in $\mathscr{Y}$. Then $M \subset X$.

Theorem 4.1. $f$ is uniformly persistent (w.r.t. $\mathscr{Y}$ ) if and only if

(1) $M$ is isolated in $X$, and

(2) $W^{s}(M) \subset \mathscr{Y}$.

Proof. The $\Rightarrow$ part is again obvious. For the converse, we first observe that Theorem 2.1 as applied to $\left.f\right|_{X}$ (and the remark following it) gives us an attractor $A$ (for $\left.f\right|_{X}$ ) dual to $M$. Since $A$ is non-empty and compact, $\mathscr{Y}$ is closed and $A \cap \mathscr{Y}=\varnothing$, we have $\eta:=d(A, \mathscr{Y})>0$. Now consider $x \in \mathscr{X} \backslash \mathscr{Y}$. Then $\Lambda^{+}(x)$ (which exists by the above assumptions) is contained in $X$ and $\Lambda^{+}(x) \not \subset M$, by (2). Since $\Lambda^{+}(x)$ is chain transitive, $\Lambda^{+}(x) \subset A$. Hence, $\liminf _{n \rightarrow \infty} d\left(f^{n}(x), \mathscr{Y}\right) \geq \eta$.

In the same way we get the following modifications of Theorems 3.1 and 3.2.

Theorem 4.2. Suppose $\left\{M_{1}, \ldots, M_{n}\right\}$ is a Morse decomposition of $M$ (under $\left.f\right|_{M}$ ) such that each $M_{i}$ is isolated in $X$. Then $f$ is uniformly persistent if and only if $W^{s}\left(M_{i}\right) \subset \mathscr{Y}$ for each $i$.

Theorem 4.3. $f$ is uniformly persistent if and only if each basic set $\Lambda$ of $\left.f\right|_{M}$ satisfies

(1) $\Lambda$ is $M$-isolated, that is, there is a neighborhood $U$ of $\Lambda$ in $X$ such that if a complete orbit $\gamma(x) \subset U$ then $\gamma(x) \subset M$, and

(2) $W^{s}(\Lambda) \subset \mathscr{Y}$. 


\section{REFERENCES}

1. E. Akin, Foundations for dynamical systems, part I, topological dynamics, preprint.

2. N. P. Bhatia and O. Hajek, Local semi-dynamical systems, Lecture Notes in Math. Vol. 90, Springer-Verlag, New York, 1969.

3. R. Bowen, $\omega$-limit sets for Axiom A diffeomorphisms, J. Diff. Eqns. 18 (1975), 333-339.

4. L. Block and J. E. Franke, The chain recurrent set, attractors and explosions, Ergod. Th. Dynam. Sys. 5 (1985), 321-327.

5. G. J. Butler, H. I. Freedman and P. Waltman, Uniformly persistent systems, Proc. Amer. Math. Soc. 96 (1986), 425-426.

6. G. J. Butler and P. Waltman, Persistence in dynamical systems, J. Diff. Eqns. 63 (1986), 255-263.

7. C. Conley, Isolated invariant sets and the Morse index, CBMS Vol. 38, Amer. Math. Soc., Providence, 1978.

8. A. Fonda, Uniformly persistent semi-dynamical systems, Proc. Amer. Math. Soc. 104 (1988), 111-116.

9. H. I. Freedman and J. W.-H. So, Persistence in discrete models of a population which may be subjected to harvesting, Nat. Res. Modeling. 2 (1987), 135-145.

10. __ Persistence in semi-dynamical systems, SIAM J. Math. Anal. 20 (1989), 930-938.

11. B. M. Garay, Uniform persistence and chain recurrence, J. Math. Anal. Appl. 139 (1989), 372-381.

12. J. K. Hale, Asymptotic behavior of dissipative systems, Math. Surveys Monographs 25, Amer. Math. Soc., Providence, 1988.

13. J. K. Hale and P. Waltman, Persistence in infinite dimensional systems, SIAM J. Math. Anal. 20 (1989), 388-395.

14. J. Hofbauer, A unified approach to persistence, Proc. Conf. Laxenburg 1987, Acta Applicandae Mathematicae, 14 (1989), 11-22.

15. J. Hofbauer, V. Hutson and W. Jansen, Coexistence for systems governed by difference equations of Lotka-Volterra type, J. Math. Biol. 25 (1987) 553-570.

16. J. Hofbauer and K. Sigmund, The theory of evolution and dynamical systems, Cambridge University Press, 1988.

17. V. Hutson and W. Moran, Persistence of species obeying difference equations, J. Math. Biol. 15 (1982) 203-213.

18. J. P. LaSalle, The stability of dynamical systems, Soc. Indust. Appl. Math., Philadelphia, 1976.

19. K. Mischaikow and R. D. Franzosa, The connection matrix theory for semiflows on (not necessarily locally compact) metric spaces, J. Diff. Eqns. 71 (1988) 270-287.

Institut für Mathematik, Universität Wien, A-1090, Vienna, Austria

Department of Mathematics, University of Alberta, Edmonton, Alberta, Canada T6G $2 \mathrm{Gl}$ 